\title{
Numerical Study for Fractional Euler-Lagrange Equations of a Harmonic Oscillator on a Moving Platform
}

\author{
D. Baleanu ${ }^{a, b}$, T. BlaszczYK ${ }^{c, *}$, J.H. Asad ${ }^{d}$ And M. Alipour ${ }^{e}$ \\ ${ }^{a}$ Department of Mathematics, Faculty of Arts and Sciences, Cankaya University, 06530 Ankara, Turkey \\ ${ }^{b}$ Institute of Space Sciences, P.O. Box, MG-23, 76900 Magurele-Bucharest, Romania \\ ${ }^{c}$ Institute of Mathematics, Częstochowa University of Technology, \\ al. Armii Krajowej 21, 42-201 Częstochowa, Poland \\ ${ }^{d}$ Department of Physics, College of Arts and Sciences, Palestine Technical University, P.O. Box 7, Tulkarm, Palestine \\ ${ }^{e}$ Department of Mathematics, Faculty of Basic Science, Babol University of Technology, \\ P.O. Box 4717-1167, Babol, Iran
}

(Received December 29, 2015; in final form June 17, 2016)

\begin{abstract}
We investigate the fractional harmonic oscillator on a moving platform. We obtained the fractional EulerLagrange equation from the derived fractional Lagrangian of the system which contains left Caputo fractional derivative. We transform the obtained differential equation of motion into a corresponding integral one and then we solve it numerically. Finally, we present many numerical simulations.
\end{abstract}

DOI: 10.12693/APhysPolA.130.688

PACS/topics: 02.60.Jh, 02.60.Nm, 02.10.Ud, 02.30.Vv

\section{Introduction}

Fractional calculus owes its origin to an old question about possibility of taking derivative to a non- integer order. It was formulated nearly at the same time as a classical calculus. Several scientists studied this issue, among them Euler, Abel, Fourier, Liouville, Riemann, Grunwald, Letnikov, and many others. Fractional calculus was regarded for a long time only from a mathematical point of view. Recently, the fractional calculus becomes a very useful and powerful tool in many branches of sciences and engineering [1-3]. Several definitions of noninteger order operators have been introduced and used; such as the Riemann-Liouville integral and differential operators, the Caputo derivatives, the Hadamard integral, the Weyl integral, etc.

The fractional variational calculus was formulated first by Riewe [4]. He got the appropriate fractional Euler-Lagrange equations, linking conservative and nonconservative cases. These fractional Euler-Lagrange equations are then used to investigate many physical problems [5-13].

Many systems in physics can be described by their Lagrangian equation, and from the Lagrangian we can obtain differential equation containing left and right fractional derivatives, these obtained equations are known as the Euler-Lagrange equations. In many cases the exact solution cannot be obtained for these equations and for this reason the numerical approach is a natural alternative to the analytical approach [14-19], and references within them.

*corresponding author; e-mail: tomasz.blaszczyk@im.pcz.pl
The rest of the paper is arranged as follows: in Sect. 2, the studied problem (harmonic oscillator on a moving platform) is discussed briefly, and some definitions of fractional operators are presented. In Sect. 3, we proposed the numerical solution of the obtained fractional Euler-Lagrange equations. Finally, the concluding remarks are presented in Sect. 4.

\section{Description of the model, and some basic definitions}

Let us study a mass-spring system with mass $m$ and spring constant $k$, included within a massless cart. This cart is travelling horizontally with a constant velocity $U$. The constant velocity $U$ is provided by an external action (i.e., see Fig. 1).

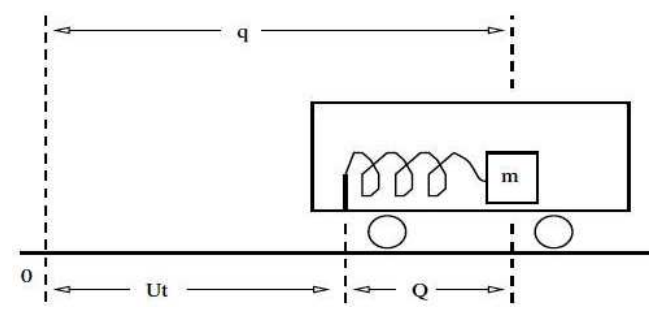

Fig. 1. Mass-spring system on a massless cart.

In this case the Lagrangian $L=T-V$ has the following form:

$$
L=\frac{1}{2} m \dot{q}^{2}-\frac{1}{2} k(q-U t)^{2} .
$$

The resulting classical Euler-Lagrange equation of motion is

$$
m \ddot{q}=-k(q-U t)
$$




$$
q(a)=\xi_{a}, \quad q(b)=\xi_{b} .
$$

If we substitute $Q=q-U t$ in Eq. (2), then we get

$$
m \ddot{Q}=-k Q
$$

with adequate boundary conditions

$$
\begin{aligned}
& Q(a)=q(a)-U a=\xi_{a}-U a, \\
& Q(b)=q(b)-U b=\xi_{b}-U b .
\end{aligned}
$$

Now, Eq. (1) can be fractionalized as

$$
L^{F}=\frac{1}{2} m\left({ }_{a}^{C} D_{t}^{\alpha} q\right)^{2}-\frac{1}{2} k(q-U t)^{2} .
$$

To obtain the fractional Euler-Lagrange equation from Eq. (6) we use results presented in [20]:

$$
\frac{\partial L^{F}}{\partial q}+{ }_{t}^{C} D_{b}^{\alpha} \frac{\partial L^{F}}{\partial_{a}^{C} D_{t}^{\alpha} q}+{ }_{a}^{C} D_{t}^{\beta} \frac{\partial L^{F}}{\partial_{t}^{C} D_{b}^{\beta} q}=0 .
$$

The obtained equation reads

$$
-k(q-U t)+m_{t}^{C} D_{b a}^{\alpha C} D_{t}^{\alpha} q=0 .
$$

Setting $Q=q-U t$ and taking its fractional derivative we have

$$
{ }_{t}^{C} D_{b a}^{\alpha C} D_{t}^{\alpha} q={ }_{t}^{C} D_{b a}^{\alpha C} D_{t}^{\alpha} Q+U_{t}^{C} D_{b a}^{\alpha C} D_{t}^{\alpha} t .
$$

So, Eq. (8) reads

or

$$
-k Q+m_{t}^{C} D_{b a}^{\alpha C} D_{t}^{\alpha} Q=-m U_{t}^{C} D_{b a}^{\alpha C} D_{t}^{\alpha} t
$$

$$
{ }_{t}^{C} D_{b a}^{\alpha C} D_{t}^{\alpha} Q-\omega Q=f_{\alpha}(t),
$$

where $f_{\alpha}(t)=-U_{t}^{C} D_{b a}^{\alpha C} D_{t}^{\alpha} t$ and $\omega=\frac{k}{m}$. Equation (11) is a non-homogeneous fractional differential equation and we aim to solve it numerically in this work. For $\alpha=1$ Eq. (11) is reduced to Eq. (4).

In fractional calculus the zeros of the fractional operators are different than the ones in classical cases. This important property generates extra term which is not present in the local classical calculus. The extra term which appears in Eq. (11) and denoted by $f_{\alpha}$ is due to the change of variables $Q=q-U t$, namely the linear
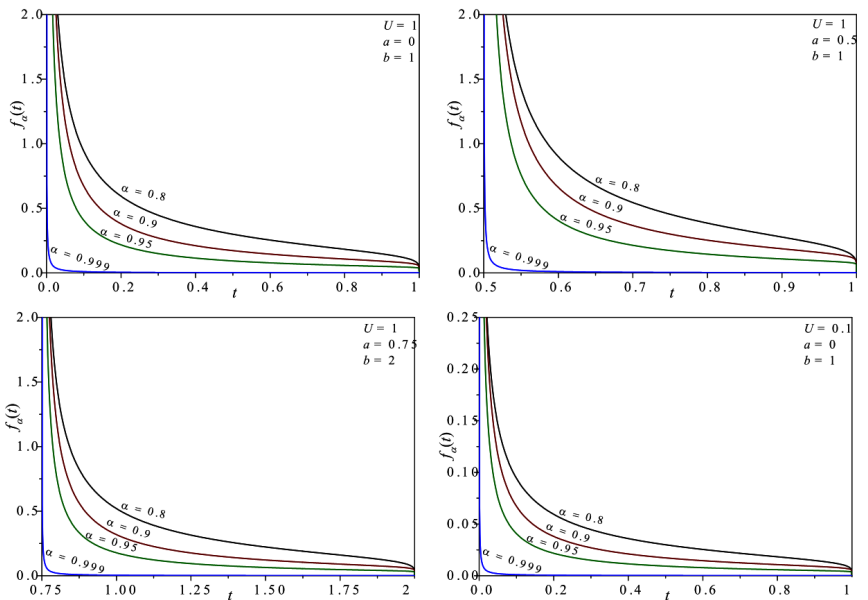

Fig. 2. The behavior of the function $f_{\alpha}$ for various parameters $U, a, b$ and order $\alpha$. term in time has a non-zero contribution which clearly shows the effect of nonlocality. In fact, the dimension of $f_{\alpha}$ is the same as of the other two terms appearing in Eq. (11). This extra term appears only when alpha is strictly between zero and one. For several values of alpha we draw three graphs (see Fig. 2) of this pure nonlocal effect in order to visualize better its behavior.

Below, we discussed the definitions of fractional integrals and derivatives [1]. These definitions are used in the following part of the paper.

The left and right Riemann-Liouville fractional integral operators are defined by:

$$
\begin{aligned}
& { }_{a} I_{t}^{\alpha} f(t)=\frac{1}{\Gamma(\alpha)} \int_{a}^{t}(t-\tau)^{\alpha-1} f(\tau) \mathrm{d} \tau, \\
& { }_{t} I_{b}^{\alpha} f(t)=\frac{1}{\Gamma(\alpha)} \int_{t}^{b}(\tau-t)^{\alpha-1} f(\tau) \mathrm{d} \tau,
\end{aligned}
$$

respectively.

The left Caputo fractional derivative (LCFD) reads

$$
\begin{aligned}
& { }_{a}^{C} D_{t}^{\alpha} f(t)= \\
& \frac{1}{\Gamma(n-\alpha)} \int_{a}^{t}(\tau-t)^{n-\alpha-1} D^{n} f(\tau) \mathrm{d} \tau .
\end{aligned}
$$

The right Caputo fractional derivative (RCFD) reads

$$
\begin{aligned}
& { }_{t}^{C} D_{b}^{\alpha} f(t)= \\
& \frac{1}{\Gamma(n-\alpha)} \int_{t}^{b}(\tau-t)^{n-\alpha-1}(-D)^{n} f(\tau) \mathrm{d} \tau,
\end{aligned}
$$

where $n-1<\alpha \leq n$ and $D^{n} \equiv \frac{\mathrm{d}^{n}}{\mathrm{~d} t^{n}}$.

\section{Numerical solution}

Here Eq. (11) is to be solved numerically, and to do this: First, we transform Eq. (11) into an integral equation. We integrate Eq. (11) twice by using the RRLFI (13) and the LRLFI (12), respectively. Finally, by using results presented in $[21,22]$ we obtain the integral form of Eq. (11)

$$
\begin{aligned}
& Q(t)-\omega\left({ }_{a} I_{t}^{\alpha}{ }_{t} I_{b}^{\alpha} Q(t)-\left.\left(\frac{t-a}{b-a}\right)^{\alpha}{ }_{a} I_{t}^{\alpha}{ }_{t} I_{b}^{\alpha} Q(t)\right|_{t=b}\right)= \\
& \quad\left(\frac{t-a}{b-a}\right)^{\alpha}\left(Q(b)-Q(a)-\left.{ }_{a} I_{t}^{\alpha}{ }_{t} I_{b}^{\alpha} f_{\alpha}(t)\right|_{t=b}\right) \\
& +{ }_{a} I_{t}^{\alpha}{ }_{t} I_{b}^{\alpha} f_{\alpha}(t)+Q(a) .
\end{aligned}
$$

Taking into account the form of the function $f_{\alpha}(t)=$ $-U_{t}^{C} D_{b a}^{\alpha C} D_{t}^{\alpha} t$ we have

$$
\begin{gathered}
Q(t)-\omega\left({ }_{a} I_{t}^{\alpha}{ }_{t} I_{b}^{\alpha} Q(t)-\left.\left(\frac{t-a}{b-a}\right)^{\alpha}{ }_{a} I_{t}^{\alpha}{ }_{t} I_{b}^{\alpha} Q(t)\right|_{t=b}\right)= \\
\left(\frac{t-a}{b-a}\right)^{\alpha}(Q(b)-Q(a)-f(b))+f(t)+Q(a),
\end{gathered}
$$

where $f(t)=U\left(-t+a+\frac{t-a}{\Gamma(2-\alpha) \Gamma(1+\alpha)}\right)$. 
Now, we introduce the following grid of nodes (with the time step $\Delta t=(b-a) / N): a=t_{0}<t_{1}<\ldots<t_{i}<$ $\ldots<t_{N}=b$, for $i=0,1, \ldots, N$.

There exist many numerical methods to obtain an approximation of integral operators of integer or noninteger order [21-25]. On the basis of discretization of fractional integrals (12) and (13) presented in our papers $[21,22,25]$ we can write the numerical scheme for the integral equation (17) as the system of $N+1$ linear equations

$$
\begin{aligned}
& Q_{i}-\omega\left(\sum_{j=0}^{i} u_{i, j} \sum_{k=j}^{N} v_{j, k} Q_{k}\right. \\
& \left.-\left(\frac{i}{N}\right)^{\alpha} \sum_{j=0}^{N} u_{N, j} \sum_{k=j}^{N} v_{j, k} Q_{k}\right)= \\
& \left(\frac{i}{N}\right)^{\alpha}\left(Q_{N}-Q_{0}-f_{N}\right)+f_{i}+Q_{0}
\end{aligned}
$$

for $i=0,1, \ldots, N$. On the basis of the numerical scheme presented above we implemented the procedure in Maple and carried out computational simulations for different values of parameters $m, k, U$ and the order $\alpha$. We applied the LU decomposition method in order to numerically solve the system (18). In all examples we assumed: $a=0, b=1$. The time domain $[0,1]$ has been divided into $N=500$ subintervals. Figure 3 showed a comparison of the solution of Eq. (4) with the solution of Eq. (11) for the order of $\alpha=1$.

Presented results demonstrate that the approximate solution has a good agreement with the exact solution.

In Fig. 4 we presented results of the numerical solutions of Eqs. (8) and (11) for various values of parameters $m$, $k, U$ and the order $\alpha$. The values of parameters used in the solution of considered equations are given in the plot legends.
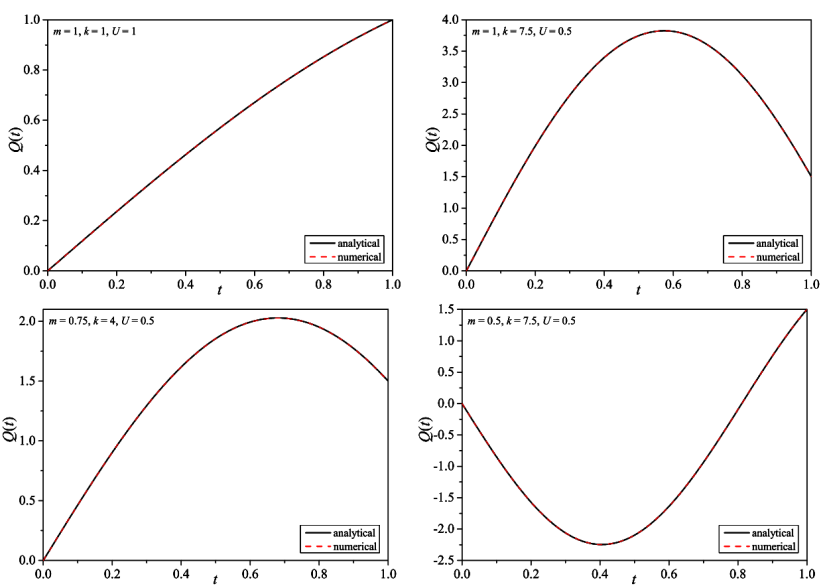

Fig. 3. Comparison of the analytical solution of Eq. (4) and the numerical solution of Eq. (11) for $\alpha=1$ and boundary conditions $\xi_{a}=0, \xi_{b}=2$.
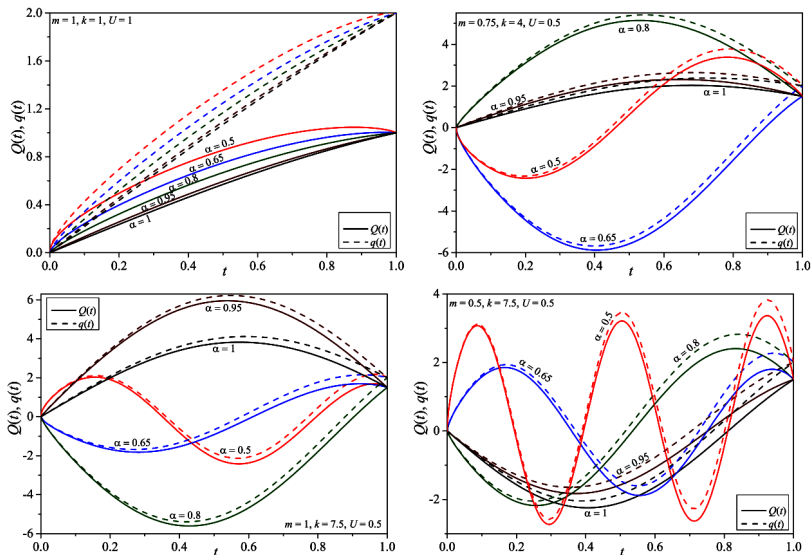

Fig. 4. Numerical solutions of Eqs. (8) (dash line) and (11) (solid line) for various values of parameters $m, k, U$ and the order $\alpha$ and boundary conditions $\xi_{a}=0, \xi_{b}=2$.
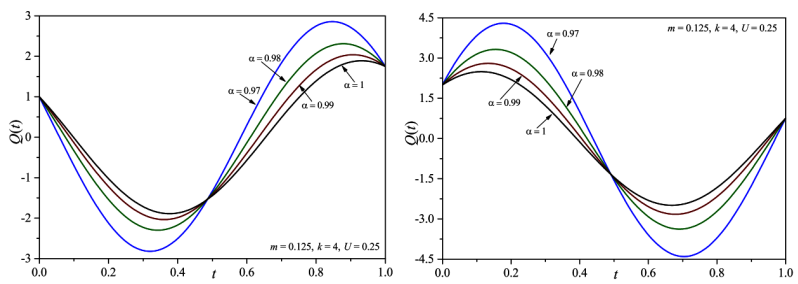

Fig. 5. Numerical solutions of Eq. (11) for various values of order $\alpha \in\{0.97 ; 0.98 ; 0.99 ; 1\}$ and boundary conditions: $\xi_{a}=1, \xi_{b}=2$ (left side) and $\xi_{a}=2, \xi_{b}=1$ (right side).
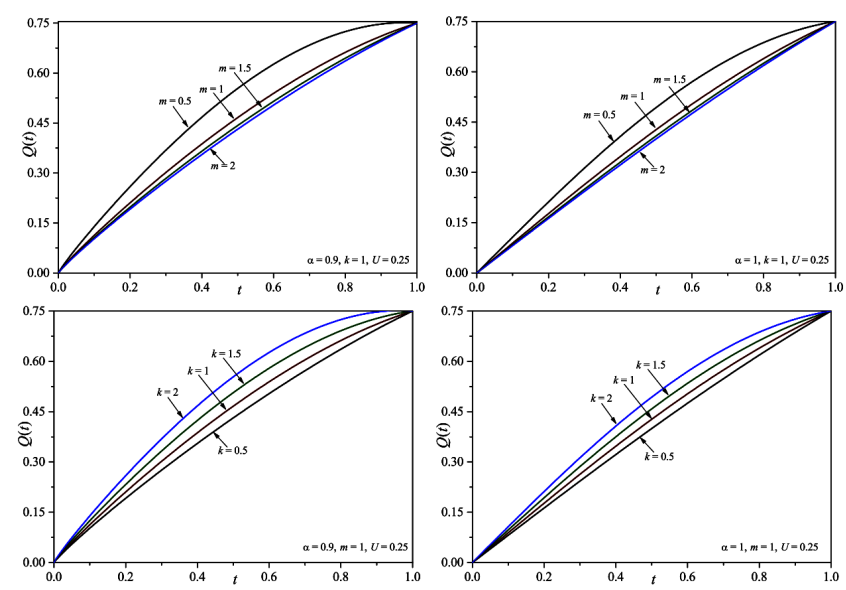

Fig. 6. Numerical solutions of Eq. (11) for various values of parameters $m, k \in\{0.5 ; 1 ; 1.5 ; 2\}$ and boundary conditions $\xi_{a}=0, \xi_{b}=1$

In Fig. 5 numerical simulations for the fixed values $m=0.125, k=4, U=0.25$ and different values of $\alpha$ are presented. In Fig. 6 we depicted the results for $\alpha=$ $0.9, \alpha=1, U=0.25$ and various values of parameters $m$ and $k$. 


\section{Conclusions}

In this paper the fractional harmonic oscillator on a moving platform was investigated. The nonhomogeneous fractional Euler-Lagrange equation involving the composition of the left and right Caputo derivatives of the order $\alpha$ was transformed into the integral form. Next, the numerical scheme for the obtained integral equation was presented. Several examples of approximate solutions of considered equation for different values of parameters $m, k, U$, and $\alpha$ was shown. The exact solution of this type of equation (except for $\alpha=1$ ) is not yet known. Our proposed numerical method of solution for $\alpha=1$ is consistent with the exact solution. One can note that by fixing the parameters and by changing $\alpha$ we get different results. On the other hand, for $\alpha$ constant and varying the mass $m$ and spring stiffness $k$ we get different behaviors of the solution. The presented results demonstrate that the proposed approach is more suitable to characterize the complex dynamics in the considered system.

\section{References}

[1] A.A. Kilbas, H.M. Srivastava, J.J. Trujillo, Theory and Applications of Fractional Differential Equations, Elsevier, Amsterdam 2006.

[2] M. Lazarevic, Advanced Topics on Applications of Fractional Calculus on Control Problems, System Stability and Modeling, WSEAS Press, 2014.

[3] W. Sumelka, T. Blaszczyk, C. Liebold, Europ. J. Mech. A/Solids 54, 243 (2015).

[4] F. Riewe, Phys. Rev. E 53, 1890 (1996).

[5] O.P. Agrawal, J. Vibrat. Contr. 13, 1217 (2007).

[6] D. Baleanu, O.P. Agrawal, Nuovo Cim. B 119, 73 (2004).

[7] D. Baleanu, T. Avkar, Czech. J. Phys. 56, 1087 (2006).
[8] M. Klimek, Czech. J. Phys. 51, 1348 (2001).

[9] M. Klimek, T. Odzijewicz, A.B. Malinowska, J. Math. Anal. Appl. 416, 402 (2014).

[10] A.B. Malinowska, T. Odzijewicz, D.F.M. Torres, Advanced Methods in the Fractional Calculus of Variations, Springer Int. Publ., London 2015.

[11] S.I. Muslih, D. Baleanu, J. Math. Anal. Appl. 304, 599 (2005).

[12] E.M. Rabei, K.I. Nawafleh, R.S. Hijjawi, S.I. Muslih, D. Baleanu, J. Math. Anal. Appl. 327, 891 (2007).

[13] F. Riewe, Phys. Rev. E 55, 3581 (1997).

[14] R. Almeida, S. Pooseh, D.F.M. Torres, Computational Methods in the Fractional Calculus of Variations, Imperial College Press, Singapore 2015.

[15] D. Baleanu, J.H. Asad, I. Petras, Commun. Theor. Phys. 61, 221 (2014).

[16] D. Baleanu, J.H. Asad, I. Petras, Nonlin. Dyn. 81, 97 (2015).

[17] T. Blaszczyk, Rom. Rep. Phys. 67, 350 (2015).

[18] P. Kumar, O.P. Agrawal, Signal Proc. 86, 2602 (2006).

[19] R. Scherer, S.L. Kalla, Y. Tang, J. Huang, Comput. Math. Appl. 62, 902 (2011).

[20] M.J. Lazo, D.F.M. Torres, J. Optim. Theory Appl. 156, 56 (2013).

[21] T. Blaszczyk, M. Ciesielski, Appl. Math. Comput. 257, 428 (2015).

[22] M. Ciesielski, T. Blaszczyk, J. Theor. Appl. Mech. 53, 959 (2015)

[23] A.P. Durajski, Eur. Phys. J. B 87, 210 (2014).

[24] A.P. Durajski, Supercond. Sci. Technol. 28, 035002 (2015).

[25] J. Siedlecki, M. Ciesielski, T. Blaszczyk, J. Appl. Math. Comput. Mech. 14, 103 (2015). 